Review began 08/09/2021 Review ended 08/21/2021 Published 08/29/2021

๑) Copyright 2021

Hatta et al. This is an open access article distributed under the terms of the Creative Commons Attribution License CC-BY 4.0., which permits unrestricted use, distribution, and reproduction in any medium, provided the original author and source are credited.

\section{Utility of Ethylene-Diamine-Tetraacetic Acid Buffer Solution With Boric Acid for Immunostaining of Specimens Stored for an Extended Period}

\author{
Hideki Hatta ${ }^{1}$, Takeshi Nishida ${ }^{1}$, Takashi Minamisaka ${ }^{1}$, Koichi Tsuneyama ${ }^{2}$, Johji Imura ${ }^{1}$ \\ 1. Department of Diagnostic Pathology, University of Toyama, Toyama, JPN 2. Department of Pathology and Laboratory \\ Medicine, Tokushima University, Tokushima, JPN
}

Corresponding author: Koichi Tsuneyama, koichi.tsuneyama@gmail.com

\begin{abstract}
Antigen modification and denaturation are recognized causes of false negatives in immunostaining. Specimens that have been stored for an extended period at room temperature show decreased immunoreactivity and may mislead the diagnosis. Studies of the molecular targeting of drugs often involve immunostaining of previous samples and, in some situations, only unstained specimens can be used. The present study aimed to develop an effective staining method to recover antigen activation in unstained specimens stored for an extended period by using ethylene-diamine-tetraacetic acid (EDTA) buffer solution with boric acid. We compared several commonly used antigen retrieval solutions and found that Tris-borateEDTA (TBE) buffer solution with a $\mathrm{pH} \geqslant 8.3$ provided sufficient antigen retrieval. However, $\mathrm{pH}$ values higher than $8.3(9.0,10.0$, and 11.0$)$ frequently caused severe tissue damage. Thus, TBE with $\mathrm{pH} 8.3$ was the most suitable antigen retrieval solution for recovering the antigenicity of specimens stored for an extended period. This procedure may allow useful immunohistochemical information, even from sections that have been stored for an extended period.
\end{abstract}

Categories: Pathology

Keywords: ethylene-diamine-tetraacetic acid, immunostaining, antigen retrieval, boric acid, old specimen, edta

\title{
Introduction
}

Antigen modification and denaturation are recognized causes of false-negative reactions in immunostaining. Specimens that have been stored for an extended period at room temperature show decreased immunoreactivity and may mislead the diagnosis [1-3]. When immunostaining is required as a companion test for the selection of cancer treatment, more than ten cut specimens must be initially prepared to avoid sample depletion. If sections stored for an extended period can also be used for immunostaining, the number of indications for companion testing will be expanded.

We previously reported new immunostaining techniques to enhance staining activity using microwave heating or ultrasonic emission [4-7]. However, the recovery of staining activity of old specimens was not easy, even in these advanced procedures. Recently, the effectiveness of a thermal treatment that uses boric acid buffer solution for DNA analysis with polymerase chain reaction (PCR) was reported, and the efficient separation performance of an ethylene-diamine-tetraacetic acid (EDTA) buffer solution containing Tris-saltbased borax has garnered attention as an electrophoresis buffer solution for DNA and RNA [8,9]. We hypothesized that this buffer might be effective in immunostaining. The present study aimed to develop an effective staining method that improves antigen activation of old unstained specimens by using an EDTA buffer solution with boric acid.

\section{Technical Report \\ Materials and Methods}

Specimens

A formalin-fixed paraffin-embedded tissue microarray block containing various autopsied organs, including breast cancer, was prepared after approval of the ethical review (No. 3972) by the ethics committee of Tokushima University Hospital. Unstained specimens can present decreased antigenicity as early as two weeks after preparation, and the decrease becomes notable after six months [1-3]. In the present study, unstained sections obtained from a tissue microarray block were left for up to eight months in the laboratory at room temperature. The control was unstained sections that were cut from the same tissue array block and used immediately. 


\section{Cureus}

Citric acid buffer solution (pH 6.0; CB6.0) is a standard antigen retrieval solution [10]. In the current study, Tris-EDTA buffer solution ( $\mathrm{pH}$ 9.0; TE9.0), boric acid buffer solution ( $\mathrm{pH} 7.4 ; \mathrm{BB} 7.4$ ), boric acid buffer solution (pH 9.0; BB9.0), and Tris-boric acid-EDTA buffer solution (pH 8.3; TBE8.3) were used.

Primary Antibodies

Mouse monoclonal antibodies against Ki-67 (neat, Nichirei Biosciences, Tokyo, Japan), p53 (neat, Nichirei Biosciences, Tokyo, Japan), ER (neat, Nichirei Biosciences, Tokyo, Japan), c-erbB2 (1:200 dilution, Nichirei Biosciences, Tokyo, Japan), and E-cadherin (neat, Nichirei Biosciences, Tokyo, Japan) were used.

Immunostaining procedure

After thermal heating using a Pascal Pressure Chamber (Agilent, Santa Clara, CA, US) in each antigen retrieval solution, a primary antibody was applied and incubated for 1 hour at room temperature [1112]. After washing with Tris-buffered saline (TBS), a dextran polymer with secondary antibodies (EnVision+ Single Reagent (HRP. Mouse), Agilent, Santa Clara, CA, US) was applied and incubated for one hour at room temperature. 3,3'-Diaminobenzidine tetrahydrochloride (DAB) was used as the substrate for color development.

Evaluation

Antigenicity recovery by each retrieval solution was evaluated using a scoring system. The degree of staining was scored as follows: staining of the control specimen $=3$, weaker than the control but positive $=2$, slightly positive $=1$, and no staining $=0$. Numerous pathologists objectively quantified the staining results and determined the stable combination of antigen activation methods.

\section{Results}

The degree of antigenicity recovery scoring is shown in Figure 1.

Ki-67

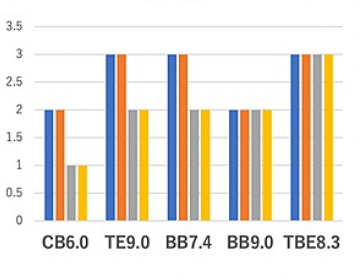

c-erbB2

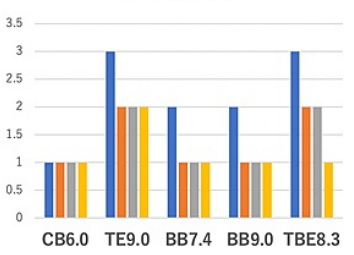

p53

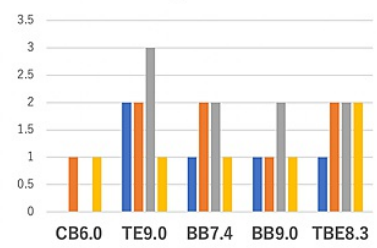

E-cadherin

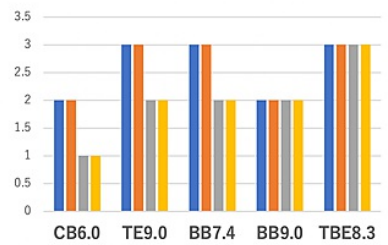

ER

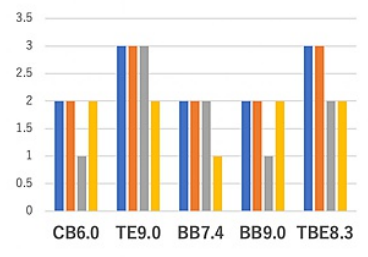

- 2 months

4 months

6 months

8 months

\section{FIGURE 1: The degree of antigenicity recovery scoring}

Antigenicity recovery by each retrieval solution was evaluated using a scoring system. The degree of staining was scored as follows: staining of the control specimen $=3$, weaker than the control but positive $=2$, slightly positive $=$ 1 , and no staining $=0$

Briefly, the order of advantages was as follows: TBE8.3 > TE9.0 $=$ BB7.4 $>$ BB9.0 > CB6.0 for Ki-67, TBE8.3 $\geqslant$ TE9.0 > BB7.4 > BB9.0 > CB6.0 for p53, TE9.0 $\geqslant$ TBE8.3 > BB7.4 $\geqslant$ BB9.0 > CB6.0 for ER, TE9.0 $\geqslant$ TBE8.3 > BB7.4 $=$ BB9.0 $>$ CB6.0 for c-erbB, and TBE8.3 $>$ TE9.0 = BB7.4 $>$ BB9.0 $>$ CB6.0 for E-cadherin. Among the buffer solutions, TBE8.3 and TE9.0 showed pronounced activation effects, and CB6.0 (which is the most commonly used buffer) displayed the lowest activation effect. We examined TBE with a pH of 9.0, 10, and 11. As expected, they showed improved activation effects, but also frequently caused severe tissue damage, such as detachment and cellular degeneration (Figure 2). 


\section{Cureus}

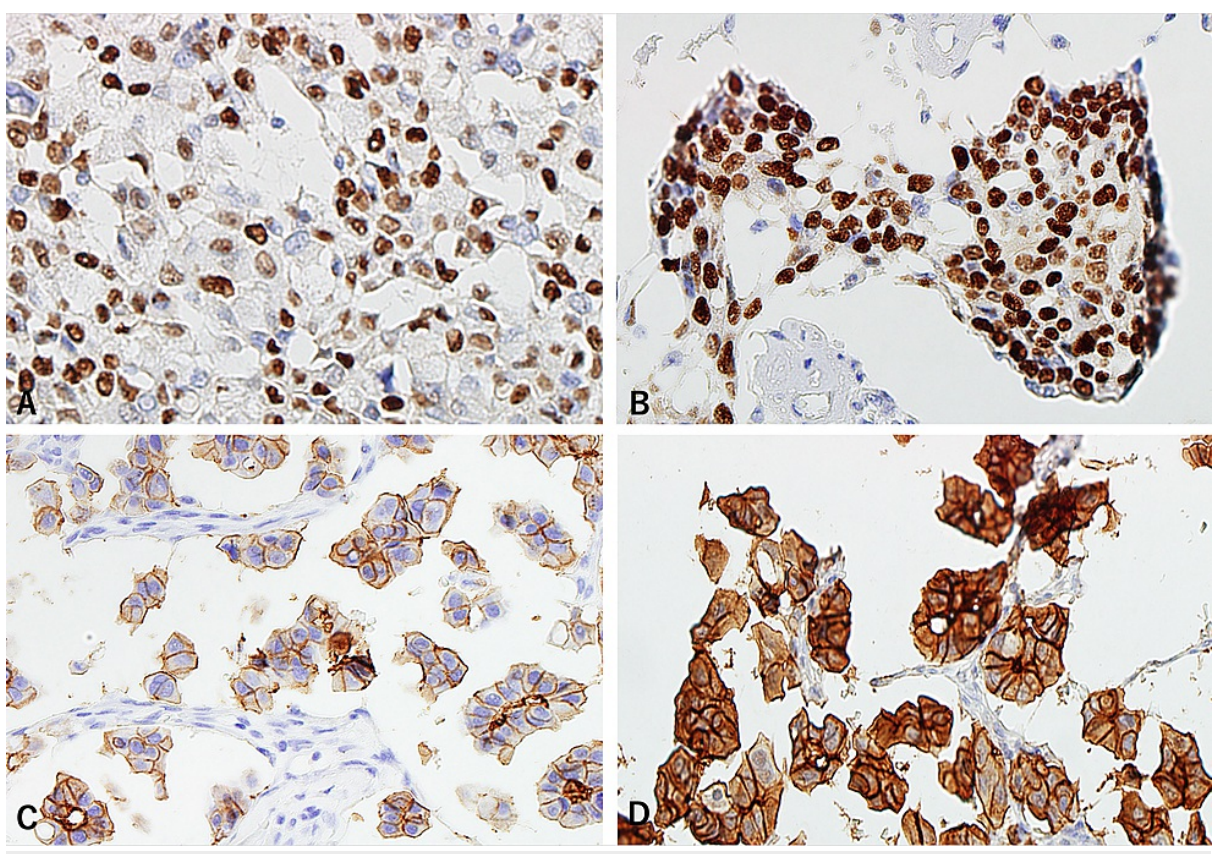

FIGURE 2: Representative staining pattern of eight-month-old specimens using solutions with $\mathrm{pH} \geq 8.3$.

Solutions used were $\mathrm{pH} \geq 8.3$. (A) ER immunostaining by TBE8.3 (Score 2); (B) ER immunostaining by TBE11 (Score 3; evident severe tissue damage); (C) Immunostaining of c-erbB2 by TBE8.3 (Score 2); (D) Immunostaining of c-erbB2 by TBE11 (Score 3; evident severe tissue damage).

Taken together, we concluded that TBE 8.3 is the most suitable antigen retrieval solution for recovering the antigenicity of sections stored for a long period at room temperature.

\section{Discussion}

The present study shows that the recovery of antigenicity can be expected using TBE 8.3 and TE9.0 for unstained specimens that have been left for up to eight months at room temperature. Although the mechanism of antigenicity masking in old specimens is complex and remains unclear, denaturation of the three-dimensional structure of the epitope of the target amino acid is one suggested reason [13-16]. Heating with TBE may help repair such a structural alteration. Here, TBE with pH exceeding 8.3 caused visible damage to specimens. There is currently no established antigen retrieval solution for specimens stored for a long time. The present results indicate the value of TBE 8.3 in the recovery of antigenicity of previously cut specimens. This antigen retrieval procedure may contribute to the additional immunohistochemical analysis of previous samples, which will be especially valuable when only sections are available.

There are several reasons for false negatives in immunostaining. A more extended fixation period or higher concentration of formalin makes immunostaining difficult [17,18]. Because the preparation of paraffin blocks varies between laboratories, standardization of immunostaining quality is a concern. In further studies, we plan to examine the antigen retrieval activity of TBE 8.3 for specimens maintained for an extended period as well as for excessively fixed specimens. The degree of antigen inactivation varies depending on the method of specimen storage, and the degree of antigen retrieval is also thought to vary depending on the method of applying heating, antibody concentration, and incubation length. In our study, TBE with $\mathrm{pH} 8.3$ was the optimal condition, but it is expected that different facilities will give different results. Based on the usefulness of TBE with $\mathrm{pH} 8.3$, it is desired that antigen activation method suitable for each facility will be examined.

\section{Conclusions}

Thermal heating using a pressure cooker in Tris-boric acid-EDTA buffer solution (pH 8.3) may allow useful immunohistochemical information, even from sections that have been stored for an extended period. If reliable immunostaining is available for sections stored for a long time, it is expected that more materials can be used for additional companion diagnoses.

\section{Additional Information}

Disclosures 
Human subjects: Consent was obtained or waived by all participants in this study. Ethics Committee of Tokushima University hospital issued approval No. 3972. Title of investigation Examination of pathological predictors and therapeutic target factors in arteriosclerosis-related organs. Animal subjects: All authors have confirmed that this study did not involve animal subjects or tissue. Conflicts of interest: In compliance with the ICMJE uniform disclosure form, all authors declare the following: Payment/services info: All authors have declared that no financial support was received from any organization for the submitted work. Financial relationships: All authors have declared that they have no financial relationships at present or within the previous three years with any organizations that might have an interest in the submitted work. Other relationships: All authors have declared that there are no other relationships or activities that could appear to have influenced the submitted work.

\section{References}

1. Bertheau P, Cazals-Hatem D, Meignin V, et al.: Variability of immunohistochemical reactivity on stored paraffin slides. J Clin Pathol. 1998, 51:370-4. 10.1136/jcp.51.5.370

2. Leong AS, Leong TY: Standardization in immunohistology. Methods Mol Biol. 2011, 724:37-68. 10.1007/978-1-61779-055-3_3

3. Wester K, Wahlund E, Sundström C, et al.: Paraffin section storage and immunohistochemistry. Effects of time, temperature, fixation, and retrieval protocol with emphasis on p53 protein and MIB1 antigen. Appl Immunohistochem Mol Morphol. 2000, 8:61-70.

4. Hatta H, Tsuneyama K, Nomoto K, et al.: A simple and rapid decalcification procedure of skeletal tissues for pathology using an ultrasonic cleaner with D-mannitol and formic acid. Acta Histochem. 2014, 116:753-7. 10.1016/j.acthis.2014.01.006

5. Hatta H, Tsuneyama K, Kondo T, Takano Y: Development of an ultrasound-emitting device for performing rapid immunostaining procedures. J Histochem Cytochem. 2010, 58:421-8. 10.1369/jhc.2010.955096

6. Hatta H, Tsuneyama K, Kumada T, et al.: Freshly prepared immune complexes with intermittent microwave irradiation result in rapid and high-quality immunostaining. Pathol Res Pract. 2006, 202:439-45. 10.1016/j.prp.2006.02.003

7. Kumada T, Tsuneyama K, Hatta H, Ishizawa S, Takano Y: Improved 1-h rapid immunostaining method using intermittent microwave irradiation: practicability based on 5 years application in Toyama Medical and Pharmaceutical University Hospital. Mod Pathol. 2004, 17:1141-9. 10.1038/modpathol.3800165

8. Akbas F, Aydin Z: Boric acid increases the expression levels of human anion exchanger genes SLC4A2 and SLC4A3. Genet Mol Res. 2012, 11:847-54. 10.4238/2012.April.3.6

9. Sanderson BA, Araki N, Lilley JL, Guerrero G, Lewis LK: Modification of gel architecture and TBE/TAE buffer composition to minimize heating during agarose gel electrophoresis. Anal Biochem. 2014, 454:44-52. 10.1016/j.ab.2014.03.003

10. Brown RW, Chirala R: Utility of microwave-citrate antigen retrieval in diagnostic immunohistochemistry . Mod Pathol. 1995, 8:515-20.

11. Hoetelmans RW, van Slooten HJ, Keijzer R, van de Velde CJ, van Dierendonck JH: Comparison of the effects of microwave heating and high pressure cooking for antigen retrieval of human and rat Bc1-2 protein in formaldehyde-fixed, paraffin-embedded sections. Biotech Histochem. 2002, 77:137-44.

12. Leong AS, Lee ES, Yin H, Kear M, Haffajee Z, Pepperall D: Superheating antigen retrieval. Appl Immunohistochem Mol Morphol. 2002, 10:263-8. 10.1097/00129039-200209000-00014

13. Leong TY, Leong AS: How does antigen retrieval work?. Adv Anat Pathol. 2007, 14:129-31. 10.1097/PAP.0b013e31803250c7

14. Robinson JM, Vandré DD: Antigen retrieval in cells and tissues: enhancement with sodium dodecyl sulfate . Histochem Cell Biol. 2001, 116:119-30. 10.1007/s004180100299

15. Ezaki T: Antigen retrieval on formaldehyde-fixed paraffin sections: its potential drawbacks and optimization for double immunostaining. Micron. 2000, 31:639-49. 10.1016/s0968-4328(99)00064-5

16. Ezaki T: Antigen retrieval: its significance and drawbacks in immunohistochemistry [Article in Japanese] . Kaibogaku Zasshi. 1996, 71:615-28.

17. Pauletti G, Dandekar S, Rong H, Ramos L, Peng H, Seshadri R, Slamon DJ: Assessment of methods for tissue-based detection of the HER-2/neu alteration in human breast cancer: a direct comparison of fluorescence in situ hybridization and immunohistochemistry. J Clin Oncol. 2000, 18:3651-64. 10.1200/JCO.2000.18.21.3651

18. Gong Y, Symmans WF, Krishnamurthy S, Patel S, Sneige N: Optimal fixation conditions for immunocytochemical analysis of estrogen receptor in cytologic specimens of breast carcinoma. Cancer. 2004, 102:34-40. 10.1002/cncr.11906 